\title{
Validation of the Sinhala version of the Pittsburgh Sleep Quality Index
}

\author{
D Anandakumar ${ }^{1}$, M Dayabandara ${ }^{1}$, S S Ratnatunga ${ }^{2}$, R Hanwella ${ }^{1}$, V A de Silva ${ }^{1}$ \\ (Index words: sleep quality, validation, Pittsburgh Sleeping Quality Index, Sri Lanka)
}

\begin{abstract}
Introduction Pittsburg Sleep Quality Index (PSQI) is a widely used standardized instrument to assess sleep quality in clinical and research settings. Objective of the study was to translate the PSQI into Sinhala language and validate using a combined qualitative and quantitative approach.

Methods Every fifth patient aged 18-60 years who attended the out-patients department of a tertiary care hospital was recruited. PSQI was translated into Sinhala using a combined qualitative and quantitative approach. Internal consistency was measured using Cronbach's alpha. Construct validity was assessed by comparing the scores in patients who were identified as having depressive disorder according to the Centre for Epidemiologic Studies Depression Scale (CES-D) and those without depressive disorder.
\end{abstract}

Results Forty-six participants with depression were compared with 159 non depressed controls. Mean PSQI component scores were significantly higher in depressed patients in 5 components. Factor analysis identified a single component explaining $53.53 \%$ of the variance. Cronbach's alpha of 0.85 indicated a high internal consistency.

Conclusions The Sinhala translation of the PSQI is a valid and reliable tool to assess sleep quality.

Ceylon Medical Journal 2016; 61: 22-25

DOI: http://doi.org/10.4038/cmj.v61i1.8255

\section{Introduction}

Medical and neurological conditions where pain is a major symptom result in poor sleep [1, 2]. It is also a feature of a wide range of psychiatric disorders such as mood disorders, psychotic disorders, dementias and anxiety disorders [3]. Often, in clinical practice, the number of hours of sleep is used as an indicator of sleep quality. Excessive daytime sleepiness resulting from poor sleep is known to cause fatigue, cognitive deficits and poor quality of life [4].

Polysomnography provides an objective measure of sleep. It is able to identify different stages of sleep. It measures the physiological parameters of sleep using electroencephalogram (EEG), electrooculogram (EOG), electrocardiogram (ECG), or electromyogram (EMG) readings. However use of a sleep laboratory in routine clinical practice is limited by the cost and inconvenience.

Therefore questionnaires which evaluate sleep have been used in routine clinical practice and research to quantify sleep quality. Sleep quality can be assessed using several indicators. These are duration of sleep, sleep latency, number of times waking up and subjective feeling of feeling refreshed on awakening and day time somnolence [5].

The Pittsburgh Sleep Quality Index (PSQI) was developed as a standardised measure of sleep quality which can be used in clinical practice [6]. The PSQI assesses sleep quality during the previous month. It consists of 19 self rated questions which are scored to obtain a total score. The 19 items are grouped into seven components which are added to give the total score. The range of scores is $0-21$. Higher scores indicate worse sleep quality. The PSQI has good internal consistency (Cronbach's alpha=0.83) [6]. It has been validated in different populations such as patients with depression, in the bereaved, elderly, and patients with anxiety disorders, chronic pain and cancers [7-9].

Since there are no sleep quality measures validated for Sri Lanka we translated the PSQI into Sinhala and validated it.

\section{Methods}

This cross sectional study was conducted in the Out Patients Department (OPD) of the National Hospital of Sri Lanka. Every fifth patient aged 18-60 years who attended

${ }^{1}$ Department of Psychiatry, Faculty of Medicine, University of Colombo and ${ }^{2}$ University Psychiatry Unit, National Hospital of Sri Lanka, Colombo, Sri Lanka.

Correspondence: MD, e-mail: <madhudayabandara@yahoo.com>. Received 9 November 2015 and revised version accepted 8 January 2016.

This is an open-access article distributed under the terms of the Creative Commons Attribution License, which permits unrestricted use, distribution, and reproduction in any medium, provided the original author and source are credited. 
the OPD was recruited until the required number was met. Repeat visits were excluded. Sample consisted of 205 patients.

The PSQI is a freely available tool for non-commercial research. It was translated into Sinhala using a combined qualitative and quantitative approach [10]. A panel of five experts who were bilingual translated the scale into Sinhala. The translations were compared and the best translation for each item of the scale was decided by consensus of the group.

The translated scale was back-translated into English by a bilingual expert who was not familiar with the original PSQI and then compared with the original scale. The translated instrument was pre-tested on a group of 10 individuals from the community.

The 19 self-rated PSQI items are added to give seven components [6]. These are subjective sleep quality, sleep latency, sleep duration, habitual sleep efficiency, sleep disturbances, use of sleep medications, and daytime dysfunction. Each component has a score that ranges from 0 to 3. All component scores are added to obtain a global score. A PSQI global score greater than 5 is indicative of significant sleep disturbance. Depressive disorder was identified using the Center for Epidemiologic Studies Depression Scale (CES-D). This scale has been validated for Sri Lanka [11]. Depressive disorder was diagnosed when the total score was $\geq 16$.

CES-D and PSQI were self administered. If the patients had difficulty understanding the questionnaire due to low literacy level or any other reason the questions were clarified by the first author. Cases were defined as participants who obtained $\geq 16$ in the CES-D and diagnosed to have depressive disorder. Participants who scored less than 16 on the CES-D were taken as controls. Written informed consent was obtained from all participants and approval was obtained from the Ethics Review Committee of the National Hospital of Sri Lanka.
Statistical analysis was done using SPSS version 18.0. Internal consistency was measured using Cronbach's alpha. Construct validity was assessed by comparing the scores in patients who were identified as having depressive disorder and those who were not. The Kolmogorov-Smirnov test showed that the scores of each PSQI component and the global PSQI score were not normally distributed. Therefore the scores for patients with and without depressive disorder were analysed using the Mann-Whitney test. The Pearson's coefficient was used to assess the correlation between the scores of the question and the PSQI scores. Principal component analysis was carried out to identify the factor structure of the scale. The Kaiser-Meyer-Olkin test (KMO) which is a measure of sampling adequacy was 0.83 and Bartlett's test of sphericity was highly significant $\left(\chi^{2}=632.69, \mathrm{df}=21\right.$, $p<0.001)$.

\section{Results}

The sample consisted of 205 patients. The mean age was 50.2 years $(\mathrm{SD}=13.5)$. One hundred and fourteen (55.6\%) were female. Forty six (22.4\%) had depressive disorder. PSQI global score ranged from 0-19. The mean PSQI global score for the total sample was 5.74. Table 1 shows the item analysis of the seven PSQI components in patients with and without depressive disorder. The means of all seven component scores were significantly higher in patients with depressive disorder. The lowest mean score was for "use of sleep medications" while the highest mean score was for "sleep latency". Table 2 shows that a single component was derived from principal component analysis. This component explained $53.53 \%$ of the variance. Table 3 shows that the corrected item-total correlation ranged from. 0.42 to 0.81 . The lowest item-total correlation was for “'use of sleep medication”. The Cronbach's alpha for the scale was 0.85 indicating high internal consistency. Deleting of any of the items did not result in increase in the Cronbach’s alpha.

Table 1. Means and standard deviations of the seven PSQI components and global score in patients with and without depressive disorder

\begin{tabular}{|c|c|c|c|}
\hline PSQI component & $\begin{array}{l}\text { Depression present }(n=46) \\
\text { Mean score for } \\
\text { item }[S D]\end{array}$ & $\begin{array}{l}\text { No depression }(n=159) \\
\text { Mean score for } \\
\text { the item }[S D]\end{array}$ & Significance \\
\hline 1. Subjective sleep quality & $1.74(1.04)$ & $0.78(0.84)$ & $\mathrm{U}=1821 ; p<0.001$ \\
\hline 2. Sleep latency & $1.98(1.18)$ & $0.93(1.13)$ & $\mathrm{U}=1996.5 ; p<0.001$ \\
\hline 3. Sleep duration & $1.85(1.14)$ & $0.87(0.98)$ & $\mathrm{U}=1963 ; p<0.001$ \\
\hline 4. Habitual sleep efficiency & $1.46(1.39)$ & $0.37(0.83)$ & $\mathrm{U}=2118.5 ; p<0.001$ \\
\hline 5. Sleep disturbances & $1.5(0.69)$ & $0.86(0.55)$ & $\mathrm{U}=1910 ; p<0.001$ \\
\hline 6. Use of sleep medications & $0.35(0.60)$ & $0.11(0.45)$ & $\mathrm{U}=2910 ; p<0.001$ \\
\hline 7. Daytime dysfunction & $1.26(1.06)$ & $0.54(0.70)$ & $\mathrm{U}=2240 ; p<0.001$ \\
\hline PSQI global Score & $10.13(5.17)$ & $4.47(3.80)$ & $\mathrm{U}=1353 ; p<0.001$ \\
\hline
\end{tabular}


Table 2. Exploratory factor analysis component matrix

\begin{tabular}{lc}
\hline PSQI component & Component 1 \\
\hline 1. Subjective sleep quality & .83 \\
2. Sleep latency & .82 \\
3. Sleep duration & .79 \\
4. Habitual sleep efficiency & .83 \\
5. Sleep disturbances & .70 \\
6. Use of sleep medications & .50 \\
7. Daytime dysfunction & .57 \\
\hline
\end{tabular}

Table 3. Reliability analysis of PSQI

\begin{tabular}{lcc}
\hline PSQI component & $\begin{array}{c}\text { Corrected item- } \\
\text { total correlation }\end{array}$ & $\begin{array}{c}\text { Cronbach's alpha } \\
\text { if item removed }\end{array}$ \\
\hline 1. Subjective sleep quality & .77 & .74 \\
2. Sleep latency & .79 & .73 \\
3. Sleep duration & .76 & .74 \\
4. Habitual sleep efficiency & .81 & .73 \\
5. Sleep disturbances & .63 & .77 \\
6. Use of sleep medications & .42 & .78 \\
7. Daytime dysfunction & .53 & .77 \\
\hline
\end{tabular}

\section{Discussion}

We found that the Sinhala translation of the PSQI was a valid and reliable instrument to assess sleep quality. It showed good construct validity by discriminating between patients with and without depression. Factor analysis identified a single component.

The initial validation study of the scale used three samples; "good sleepers" who were healthy controls, "poor sleepers" consisting of patients with major depressive disorder and a third clinical sample of "poor sleepers" referred for evaluation [6]. The study reported distinctive component and global score profiles in these groups. Since poor sleep is a core feature of depressive disorder, we used patients with depressive disorder as the "poor sleeper" group. We found that the PSQI, based on the total score, was able to differentiate between depressed and non depressed groups.

The original validation study reported a Chronbach's alpha of 0.83 which is similar to our sample [6]. This indicates that the items measure the same construct. Itemtotal correlation found that the lowest correlation was for "day time dysfunction" and "use of sleep medication". This is similar to findings from other studies [7].

Exploratory factor analysis identified a single factor. Factor analytical studies of the PSQI have mostly found a single factor although some studies have reported a three factor model [12-15]. In many studies "use of sleep medications" did not load well into the one factor model. In our study too "use of sleep medications" had the lowest factor loading (0.5).

PSQI has been translated into 56 different languages. It has been validated in many clinical populations. It has been validated in patients with psychiatric illness as well as physical illness. The PSQI can be used to assess sleep quality in research studies. It can also be used in routine clinical practice as the tool is easy to administer. It can be used as a screener to identify patients who need sleep studies. It can also be used to monitor progress of interventions. Although it can be used as a selfadministered tool some patients may need help to complete the scale.

There were several limitations in our study. We did not assess test-retest reliability. We used two groups with and without depressive disorder to establish construct validity. Our sample of "good sleepers" were people presenting to the OPD with symptoms but without complaints about sleep. A healthy control group would have been a better comparison group. Although a similar method has been used in many studies, polysomnography would have been a more objective measure.

\section{Conflicts of interests}

There are no conflicts of interest.

\section{References}

1. Purabdollah M, Lakdizaji S, Rahmani A, Hajalilu M, Ansarin K. Relationship between Sleep Disorders, Pain and Quality of Life in Patients with Rheumatoid Arthritis. J Caring Sci 2015; 4: 233-41.

2. Roth T. The effect of comorbid psychiatric and medical illnesses on sleep disorder treatment. J Clin Psychiatry 2015; 76: e1146.

3. American Psychiatric Association DSM-IV. Diagnostic and Statistical Manual of Mental Disorders. $4^{\text {th }}$ edition. Washington, DC: American PsychiatricAssociation 1994.

4. Roth T. Effects of excessive daytime sleepiness and fatigue on overall health and cognitive function. J Clin Psychiatry 2015; 76: e1145.

5. Rener-Sitar K, John MT, Bandyopadhyay D, Howell MJ, Schiffman EL. Exploration of dimensionality and psychometric properties of the Pittsburgh Sleep Quality Index in cases with temporomandibular disorders. Health Qual Life Outcomes 2014; 12: 10.

6. Buysse DJ, Reynolds CF, Monk TH, Berman SR, Kupfer DJ. The Pittsburgh Sleep Quality Index: a new instrument for psychiatric practice and research. Psychiatry Res 1989; 28: 193-213.

7. Beck SL, Schwartz AL, Towsley G, Dudley W, Barsevick 
A. Psychometric evaluation of the Pittsburgh Sleep Quality Index in cancer patients. J Pain Symptom Manage 2004; 27 : 140-8.

8. Bush AL, Armento ME, Weiss BJ, et al. The Pittsburgh Sleep Quality Index in older primary care patients with generalized anxiety disorder: psychometrics and outcomes following cognitive behavioral therapy. Psychiatry Res 2012; 199: $24-30$.

9. Carpenter JS, Andrykowski MA. Psychometric evaluation of the Pittsburgh Sleep Quality Index. J Psychosom Res 1998; 45: 5-13.

10. Sumathipala A, Murray J. New approach to translating instruments for cross-cultural research: a combined qualitative and quantitative approach for translation and consensus generation. Int J Methods Psychiatr Res 2000; 9: 87-95.

11. de Silva VA, Ekanayake S, Hanwella R. Validity of the Sinhala version of the centre for epidemiological studies depression scale (CES-D) in out-patients. Ceylon Med J 2014; 59: 8-12.

12. Mollayeva T, Thurairajah P, Burton K, et al. The Pittsburgh sleep quality index as a screening tool for sleep dysfunction in clinical and non-clinical samples: A systematic review and meta-analysis. Sleep Med Rev 2015; pii: S1087-0792 (15)00021-0.

13. Zhong QY, Gelaye B, Sanchez SE, Williams MA. Psychometric Properties of the Pittsburgh Sleep Quality Index (PSQI) in a Cohort of Peruvian Pregnant Women. $J$ Clin Sleep Med 2015; 11: 869-77.

14. Ho RT, Fong TC. Factor structure of the Chinese version of the Pittsburgh sleep quality index in breast cancer patients. Sleep Med 2014; 15: 565-9.

15. Tomfohr LM, Schweizer CA, Dimsdale JE, Loredo JS. Psychometric characteristics of the Pittsburgh Sleep Quality Index in English speaking non-Hispanic whites and English and Spanish speaking Hispanics of Mexican descent. J Clin Sleep Med 2013; 9: 61-6. 